thrombolysis in one patient (3\%). Successful revascularization was achieved in 31 patients (94\%), including TICI III in 16 patients $(48.5 \%)$. The average number of passes to reperfusion was 1.6 (95\% CI 1.3-2.0). Mean time to reperfusion was 47 minutes. Symptomatic intraparenchymal hemorrhage following reperfusion occurred in 2 patients (6\%). Procedural complications occurred in 4 patients (12\%), including dissection, microwire perforation, and hemorrhage into a pre-existing intracranial tumor; all complications were unrelated to the SOFIA. Mortality was $21.2 \%$ secondary to failed revascularization, hemorrhagic transformation, and severe baseline medical disease.

Conclusion SOFIA is a safe and effective intermediate catheter for mechanical and aspiration thrombectomy in acute stroke intervention. In conjunction with stent retrievers and suction aspiration technique, successful revascularization was $94 \%$, with average number of passes of 1.6. There were no complications directly attributable to the use of SOFIA.

Disclosures J. Wong: None. H. Do: 2; C; MicroVention, Inc. N. Telischak: None. A. Moraff: None. M. Marks: None. R. Dodd: None. J. Heit: 2; C; MicroVention, Inc.

\section{E-012 COILS COATED WITH A STATIN ACCELERATE INTRA- ANEURYSMAL ORGANIZATION}

T Kodama. Neurosurgery, The Jikei University School of Medicine, Tokyo, Japan

\subsection{6/neurintsurg-2016-012589.84}

The safety and efficacy of endovascular coil embolization of intracranial aneurysms is widely accepted. However, recurrence of aneurysms in large cases after coil embolization is a serious problem which remains to be solved. A major cause of recurrence is incomplete organization within the aneurysm. We have been developing modified coils. We read these two papers, and we found the statin reduces aneurymal size. (Am J Cardiol. 1996;97:279-280. Eur J Vasc Endovasc Surg. 2006;32:21-26.) If oral administration of a statin reduces aneurysm growth, could coils coated with a statin be more effective? This discovery was what motivated us to conduct this study. We ligated the distal External carotid artery and made an experimental aneurysm. We then inserted a coil coated with a statin to be reexamined after two and four weeks. We removed at 2 and 4 weeks after coils were implanted. In the case of implanted simvastatin coils, thick tissue had formed at the end of the coils and acted as a plug to inhibit blood flowing into the space of an aneurysm. On the other hand, in the case of the unmodified coil, blood had flowed into the space where the coil was lodged. We analyzed histochemical staining. We found endothelialization at the orifice of an experimental aneurysm, and we saw that there was proliferation of smooth muscle cells within the aneurysm. The percent of organized area of the simvastatin group was significantly higher than the unmodified coil group. We tested another 6 statins in the same way. Two weeks after coil implantation, the experimental aneurysm was cut vertically. The Aneurysmal cavity was fully filled with cellular tissue in the almost cases. In the arterial bifurcation, hemodynamic stress causes wall shear stress, endothelial dysfunction, imbalance of NO, and vascular inflammation. Vascular inflammation causes the infiltration of macrophage, increase of MMP, and destruction of ECM, Or migration of SMC. What are the effect of a statin? We suggest 4 points. First improving endothelial function, second decreasing oxidative stress, third decreasing the vascular inflammation. And fourth statin suppresses the excretion of MMP and inhibits the destruction of ECM. Coils coated with simvastatin effectively accelerated intra-aneurysmal organization and endothelialization over the coils at the orifice to ECA sacs in a rat aneurysm model. Simvastatin is widely used to lower cholesterol, so its safety is already established. We are now preparing drug-eluting coils to optimize the local concentration of simvastatin and its duration of efficacy.

Disclosures T. Kodama: None.

\section{E-013 HEAD AND NECK ENDOVASCULAR REPAIR OF VASCULAR MALFORMATIONS}

W Yakes. Vascular Malformation Center, Englewood, CO

\subsection{6/neurintsurg-2016-012589.85}

Purpose To determine the efficacy of ethanol embolotherapy of extracranial head and neck vascular malformations of all types, particularly after failure of other endovascular and surgical treatments.

Materials and methods One hundred and sixty-six patients (64 males, 102 females; mean age: 38 yrs) presented with extracranial arteriovenous malformations (AVMs) of the head and neck area. Over half of the patients had undergone previous failed therapies. All patients underwent ethanol embolotherapy under general anesthesia. Forty-five patients had AVMs and 121 patients had venous malformations (VM).

Results Of 45 AVM patients, 26 patients are cured (mean follow-up $2 \frac{1}{2}$ years); of 121 venous malformation patients, 65 are at end-therapy (mean follow-up $4 \frac{1}{2}$ years). The remaining patients are not at end-therapy and are being treated for their residual malformations. In AVM follow-up, arteriography is the main imaging modality to determine cure or residual AVM as MR is less sensitive in the evaluation of residual AVM. In VM follow-up, MR is the main imaging tool, particularly with T-2 fat suppression and/or STIR imaging. All patients demonstrated improvement post-therapy. Complications were $4.5 \%$, to include bleeding (self-limited), partial $7^{\text {th }}$ nerve palsy (with recovery), skin injury (not requiring skin grafts), infection, and pain.

Conclusions Ethanol has proven its consistent curative potential at long-term follow-up for high-flow AVMs and low-flow $\mathrm{VM}$ lesions at long-term follow-up as lesions in the periphery. Complication rates remain low. The procedures are tolerated well by the patients and done on an out-patient basis. Prior surgery and embolization procedures can cause difficulty in lesion access, but does not obviate further ethanol endovascular treatment.

Disclosures W. Yakes: None.

\section{E-014 MANAGEMENT OF BLOOD PRESSURE FOR ACUTE ISCHEMIC STROKE IN THE MODERN ERA OF MECHANICAL THROMBECTOMY}

S Mannava, A Garg. Neurology, University of Oklahoma, Oklahoma City, OK

\subsection{6/neurintsurg-2016-012589.86}

Background Five recent trials provided level 1 evidence for the benefit of mechanical thrombectomy in select patients 